\title{
The role of diffusion tensor imaging of the liver in children with autoimmune hepatitis
}

\author{
Ahmed Abdel Khalek Abdel Razek ${ }^{1 A, E, F}$, Ahmed Abdalla ${ }^{2 A, D}$, Ahmed Megahed ${ }^{2 B, C}$, Mohamed Elsayed Ahmed ${ }^{2 C, D}$, \\ Suzy Abd ElMabood ${ }^{\text {2C,D,E, }}$, Rihame Abdel Wahab ${ }^{10, E, F}$
}

'Department of Diagnostic Radiology, Mansoura Faculty of Medicine, Mansoura University, Mansoura, Egypt

2Department of Paediatrics, Paediatric University Hospital, Mansoura Faculty of Medicine, Mansoura University, Mansoura, Egypt

\section{Abstract}

Purpose: To evaluate the role of diffusion tensor imaging (DTI) of the liver in children with autoimmune hepatitis (AIH).

Material and methods: A prospective study was done on 42 children with AIH (30 girls and 12 boys, with a mean age of 13 years) and 20 age- and sex-matched healthy control children. They underwent DTI of the liver and laboratory tests. Liver biopsy was done for the patients. The mean diffusivity (MD) and fractional anisotropy (FA) of the liver were calculated and correlated with the pathological results.

Results: The mean MD and FA of the liver in children with AIH were $1.42 \pm 0.06 \times 10^{-3} \mathrm{~mm}^{2} / \mathrm{s}$ and $0.37 \pm 0.11$; and in the control children they were $1.55 \pm 0.07 \times 10^{-3} \mathrm{~mm}^{2} / \mathrm{s}$ and $0.25 \pm 0.03$, respectively. The MD and FA were significantly different in the children with AIH compared to the control children $(p=0.001)$. The cutoff MD and FA used to differentiate patients from controls were $1.50 \times 10^{-3} \mathrm{~mm}^{2} / \mathrm{s}, 0.31$ with AUC of 0.919 and 0.813 , sensitivity of $97.6 \%$ and $66.7 \%$, a specificity of $80 \%$ and $70 \%$, an accuracy of $94.2 \%$ and $67.3 \%$, PPV of 95.3 and 90.3 , and NPV of 88.9 and 33.3, respectively. There was significantly lower MD and higher FA of the liver in children with AIH type I $(n=31)$ than type II $(n=11)(p=0.001)$, and patients with $(n=9)$ and without $(n=33)$ overlap syndrome $(p=0.005)$.

Conclusions: We concluded that DTI parameters can help to diagnose AIH, detect its phenotyping, and give clues as to the presence of associated overlap syndrome.

Key words: autoimmune hepatitis, diffusion, MR imaging, hepatic, fibrosis.

\section{Introduction}

Autoimmune hepatitis (AIH) is a chronic autoimmune disease with progressive necro-inflammatory liver disease that presents with clinical presentation varying from minor symptoms to acute liver failure. AIH is characterized biochemically by high level of transaminase, histologically by the presence of interface hepatitis, and serologically by a high level of immunoglobulin G (IgG) [1-4]. AIH is further subdivided into type I and type II, depending on the autoantibody profile: AIH type I is characte- rized by positivity for the anti-nuclear antibody (ANA) and/or anti-smooth muscle antibody (ASMA), and AIH type II is characterized by the presence of anti-liver kidney microsomal antibody type I (anti-LKM1) and/or anti-liver cytosol type 1 [5-9]. Overlap syndrome describes variant forms of AIH, which includes features of a combination of $\mathrm{AIH}$, primary sclerosing cholangitis, and primary biliary cirrhosis. These disorders may present simultaneously or consecutively. AIH generally responds to immunosuppressive treatment. Liver biopsy remains the reference standard for the diagnosis and staging of AIH, but it is an invasive

Correspondence address:

Ahmed Abdel Khalek Abdel Razek MD, Department of Diagnostic Radiology, Mansoura Faculty of Medicine, Elgomheryia Street, Mansoura, Egypt 3512, phone: 00201061948567, fax: 0020502259146, e-mail: arazek@mans.edu.eg

Authors' contribution:

A Study design · B Data collection · C Statistical analysis · D Data interpretation · E Manuscript preparation · F Literature search · G Funds collection 
procedure with risk of complications [3-7]. Limited studies discuss the role of shear-wave elastography, magnetic resonance (MR) elastography, and MR spectroscopy in the diagnosis and monitoring of patients with $\mathrm{AIH}$, but these results are preliminary and overlapping [10-13].

Diffusion tensor imaging (DTI) provides in-vivo tissue microstructure information by measuring the water diffusivity for a given voxel that can be represented by parameters such as the mean diffusivity (MD) and the fractional anisotropy (FA) [14-21]. Some studies have reported that DWI is a promising technique in the detection and quantification of hepatic inflammation and fibrosis in adults and children [22-24]. Some studies also discuss the role of DTI in the detection of fibrosis in hepatic fibrosis and biliary atresia [25-29]. One study reported that FA is more sensitive than $\mathrm{MD}$ for the diagnosis of mild-to-moderate liver fibrosis [25], and another study added that MD is helpful in detecting liver fibrosis but cannot differentiate different grades of hepatic fibrosis in neonates with biliary atresia [26]. Another recent study added that the diagnostic performance of MD 2 using only $2 b$-values showed excellently diagnostic performance in the diagnosis of hepatic fibrosis [27]. To our knowledge, there is no previous literature discussing the role of DTI of the liver in children with AIH.

The aim of this study is to evaluate the role of DTI of the liver in children with AIH.

\section{Material and methods}

\section{Patients}

Informed consent from the parents of paediatric patients and controls and institutional review board approval were obtained. A prospective study was conducted on 44 successive untreated children with pathologically proven $\mathrm{AIH}$ according to Simplified Criteria for the Diagnosis of AIH in Children [9]. Two patients were excluded from the study because they did not complete the MR imaging. The final patients included 42 children ( 30 girls and 12 boys, with

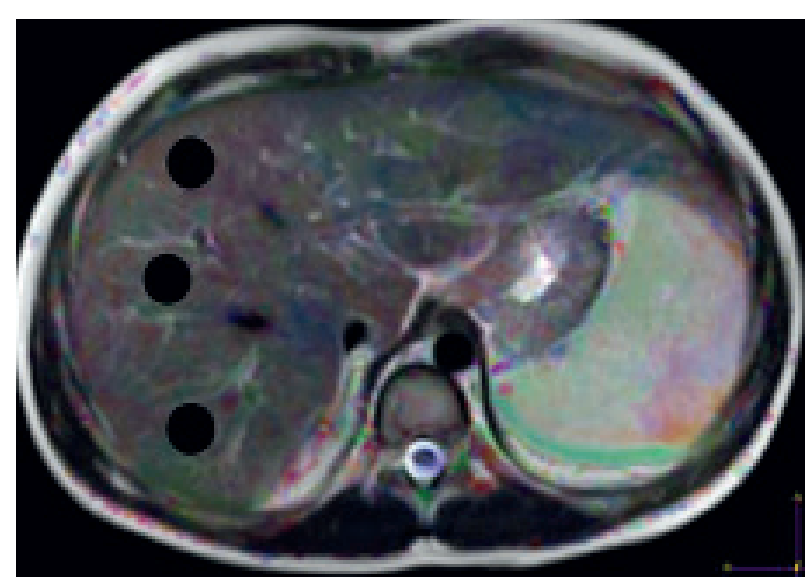

Figure 1. Regions of interest localization of the liver: colour FA map of the liver shows the localization of the 3 regions of interest (ROI) within the hepatic parenchyma age range 8 - 16 years; mean age 13 years). Twenty age- and sex-matched healthy children (12 girls and 8 boys, with age range $7-16$ years; mean age 12 years) were included as a control group. The children in the control group had no previous medical history or current liver disease and had normal liver function tests. DTI of the liver and laboratory tests (ANA, ASMA, and anti-LKM1) were done for patients and controls, and liver biopsy was done only for patients. The patients were AIH type I $(n=31)$ and type II $(n=11)$, and patients were with $(n=9)$ and without $(n=33)$ overlap syndrome.

\section{Magnetic resonance imaging}

MR imaging was done on a $1.5 \mathrm{~T}$ scanner (Ingenia, Philips, Philips Medical Systems, Best, Netherlands). Patients fasted for 4-6 hours prior to the study, and sedation was administered for children 7-10 years old, who were afraid of MR and to avoid motion artifacts $(n=25)$ using oral chloral hydrate (70-80 $\mathrm{mg} / \mathrm{kg}$ body weight), and children older than 10 years $(n=39)$ were scanned without sedation. Patients and controls underwent axial T2-weighted Fast Recovery Fast Spin Echo sequence (TR/ $\mathrm{TE}=3200 / 110 \mathrm{~ms})$ and T1-weighted images $(\mathrm{TR} / \mathrm{TE}=$ $600 / 25 \mathrm{~ms}$ ) of the liver. The scanning parameters were as follows: field of view $(\mathrm{FOV})=22-25 \mathrm{~cm}$, section thickness $=7 \mathrm{~mm}$, inter-slice gap $=1 \mathrm{~mm}$, and data matrix $=$ $256 \times 224$. DTI of the liver was done using a single-shot echo-planar imaging sequence $(\mathrm{TR} / \mathrm{TE}=3200 / 90 \mathrm{~ms})$ with parallel imaging, automatic shimming, and chemical shift selective fat suppression technique. Diffusion gradients were applied along 32 axes, using a $b$-value of 0 and $1000 \mathrm{~s} / \mathrm{mm}^{-2}$. The scanning parameters were FOV $=22 \times$ $25 \mathrm{~cm}$, slice thickness $=7 \mathrm{~mm}$, inter-slice gap $20 \%$, data matrix $=192 \times 154$, and the total scan time was 7-8 min.

\section{Image analysis}

Image analysis was performed by one radiologist (AR), an expert in MR imaging, with 15 years of experience, who was blinded to the clinical presentation and pathological results. The images were loaded into a DTI software workstation (View Forum 7.2.0.1 exported patient image data, Philips Medical System, Best, Netherlands), and automated registration of DTI data was performed. A circular region of interest (ROI) measuring $3-4 \mathrm{~cm}^{2}$ was placed on the colour FA map at 3 different regions of the hepatic parenchyma, on 3 consecutive slices, away from the vascular and biliary structures (Figure 1). The mean of these 9 values was calculated, which represents the MD and FA of each patient used for statistical analysis.

\section{Statistical analysis}

The statistical analysis of data was done using Statistical Package for the Social Sciences (SPSS, Inc., Chicago, IL, 
USA) software, version 21. The analysis of the data was done to test a statistically significant difference between groups. Student's $t$-test was used to compare 2 groups, and the one-way ANOVA test was used to compare more than 2 groups. The receiver operating characteristic (ROC) curve was plotted for calculation of the area under the curve (AUC). The cut-off point was chosen at the highest accuracy. The sensitivity, specificity, and accuracy were calculated. The $p$ value was considered significant if less than or equal to 0.05 at a confidence interval of $95 \%$.

\section{Results}

The mean age in the study group with AIH was 13 years, and AIH was more common in females. Table 1 shows the laboratory autoantibodies and immunoglobulin in patients with AIH. There was a significantly higher level of $\operatorname{IgG}(p=0.001)$ and ASMA $(p=0.032)$ and an insignificant difference in ANA $(p=0.34)$ and anti-LKM1 $(p=0.08)$ between patients and controls (Table 1$)$.

The mean MD and FA of the liver in $\mathrm{AIH}$ was 1.42 $\pm 0.06 \times 10^{-3} \mathrm{~mm}^{2} / \mathrm{s}$ and $0.37 \pm 0.11$, and in the control group it was $1.55 \pm 0.07 \times 10^{-3} \mathrm{~mm}^{2} / \mathrm{s}$ and $0.25 \pm 0.03$, respectively. The MD was significantly lower and the FA was significantly higher in children with AIH compared to the controls $(p=0.001)$ (Table 2). The cutoff MD and FA used to differentiate patients from controls were 1.50 $\times 10^{-3} \mathrm{~mm}^{2} / \mathrm{s}$ and 0.31 with AUC of 0.919 and 0.813 , sensitivity of $97.6 \%$ and $66.7 \%$, specificity of $80 \%$ and $70 \%$, and an accuracy of $94.2 \%$ and $67.3 \%$, respectively (Table 3, Figure 2).

The mean MD and FA of the liver in AIH type I $(n=31)$ was $1.44 \pm 0.04 \times 10^{-3} \mathrm{~mm}^{2} / \mathrm{s}$ and $0.33 \pm 0.09$, and in AIH type II $(n=11)$ it was $1.34 \pm 0.06 \times 10^{-3} \mathrm{~mm}^{2} / \mathrm{s}$ and 0.50 \pm 0.03 , respectively. The MD was significantly lower and the FA was significantly higher in the children with AIH type II compared to type I $(p=0.001)$ (Table 2$)$. The cutoff $\mathrm{MD}$ and FA used to differentiate AIH type I from type II were $1.42 \times 10^{-3} \mathrm{~mm}^{2} / \mathrm{s}$ and 0.42 with an AUC of 0.915 and 0.959 , sensitivity of $90.9 \%$ and $90.9 \%$, specificity of $74.2 \%$ and $77.4 \%$, and an accuracy of $78.6 \%$ and $80.9 \%$ of both reviewers, respectively (Table 3, Figure 3).

The mean MD and FA of the liver in AIH without overlap syndrome $(n=33)$ was $1.43 \pm 0.06 \times 10^{-3} \mathrm{~mm}^{2} / \mathrm{s}$ and $0.33 \pm 0.09$, and in AIH with overlap syndrome $(n=9)$ it was $1.36 \pm 0.05 \times 10^{-3} \mathrm{~mm}^{2} / \mathrm{s}$ and $0.51 \pm 0.003$, respectively. The MD was significantly lower and the FA was significantly higher in the children with AIH with overlap syndrome compared to those with AIH without overlap syndrome $(p=0.005)$ (Table 2$)$. The cutoff MD and FA used to differentiate AIH with overlap syndrome from AIH without overlap syndrome were $1.42 \times 10^{-3}$ $\mathrm{mm}^{2} / \mathrm{s}$ and 0.50 with AUC of 0.828 and 0.963 , sensitivity of $88.9 \%$ and $88.9 \%$, specificity of $69.7 \%$ and $93.9 \%$, and accuracy of $73.8 \%$ and $92.9 \%$, respectively (Table 3, Figure 4).
Table 1. Laboratory findings of patients with autoimmune hepatitis and controls

\begin{tabular}{|l|c|c|c|}
\hline Factor & $\begin{array}{c}\text { Patients } \\
(n=42)\end{array}$ & $\begin{array}{c}\text { Controls } \\
(n=20)\end{array}$ & $p$-value \\
\hline $\lg G$ & $3002(1160-5869)$ & $826(570-1233)$ & 0.001 \\
\hline ASMA & $11.5(6-152)$ & $8.5(3-15)$ & 0.032 \\
\hline ANA & $10.0(5-92)$ & $9(3-17)$ & 0.34 \\
\hline Anti-LKM1 & $8.0(5-44)$ & - & 0.08 \\
\hline
\end{tabular}

Table 2. The mean, $M D\left(\times 10^{-3} \mathrm{~mm}^{2} / \mathrm{s}\right)$ and FA of patients and controls

\begin{tabular}{|l|c|c|c|c|}
\hline Factor & MD & $p$-value & FA & $p$-value \\
\hline Patients $(n=42)$ & $1.42 \pm 0.06$ & 0.001 & $0.37 \pm 0.11$ & 0.001 \\
\hline Controls $(n=20)$ & $1.55 \pm 0.07$ & & $0.25 \pm 0.03$ & \\
\hline Type I $(n=31)$ & $1.44 \pm 0.04$ & 0.001 & $0.33 \pm 0.09$ & 0.001 \\
\hline Type II $(n=11)$ & $1.34 \pm 0.06$ & & $0.50 \pm 0.03$ & \\
\hline No overlap $(n=33)$ & $1.43 \pm 0.06$ & 0.005 & $0.33 \pm 0.09$ & 0.001 \\
\hline Overlap $(n=9)$ & $1.36 \pm 0.05$ & & $0.51 \pm 0.00$ & \\
\hline
\end{tabular}

MD - mean diffusivity, FA - fractional anisotropy

Table 3. The ROC curve results of $M D\left(\times 10^{-3} \mathrm{~mm}^{2} / \mathrm{s}\right)$ and FA of patients vs. controls

\begin{tabular}{|c|c|c|c|c|c|}
\hline Factor & AUC & $\begin{array}{c}\text { Cut-off } \\
\text { point }\end{array}$ & Sensitivity & Specificity & Accuracy \\
\hline \multicolumn{6}{|c|}{ Patients vs. controls } \\
\hline $\mathrm{MD}$ & 0.919 & 1.50 & 97.6 & 80.0 & 94.2 \\
\hline FA & 0.813 & 0.31 & 66.7 & 70.0 & 67.3 \\
\hline \multicolumn{6}{|c|}{ Type I vs. type II } \\
\hline MD & 0.915 & 1.42 & 90.9 & 74.2 & 78.6 \\
\hline $\mathrm{FA}$ & 0.959 & 0.42 & 90.9 & 77.4 & 80.9 \\
\hline \multicolumn{6}{|c|}{ Overlap vs. no overlap } \\
\hline $\mathrm{MD}$ & 0.828 & 1.42 & 88.9 & 69.7 & 73.8 \\
\hline $\mathrm{FA}$ & 0.963 & 0.50 & 88.9 & 93.9 & 92.9 \\
\hline
\end{tabular}

MD - mean diffusivity, FA - fractional anisotropy

\section{Discussion}

The main findings in our work are the significantly lower $\mathrm{MD}$ and higher FA of the liver in patients with AIH than in the controls. There was significant lower MD and higher FA of the liver in children with AIH type II compared to children with AIH type I, and patients with overlap syndrome compared to patients without the syndrome.

In this work, AIH was more common in females in the $2^{\text {nd }}$ decade of life with a significantly higher level of the $\mathrm{IgG}$ and ANA. Previous studies reported that AIH is more common in females in the $2^{\text {nd }}$ and $3^{\text {rd }}$ decades, characterized in the laboratory by elevated autoimmune antibodies and immunoglobulin, histologically by the presence of 

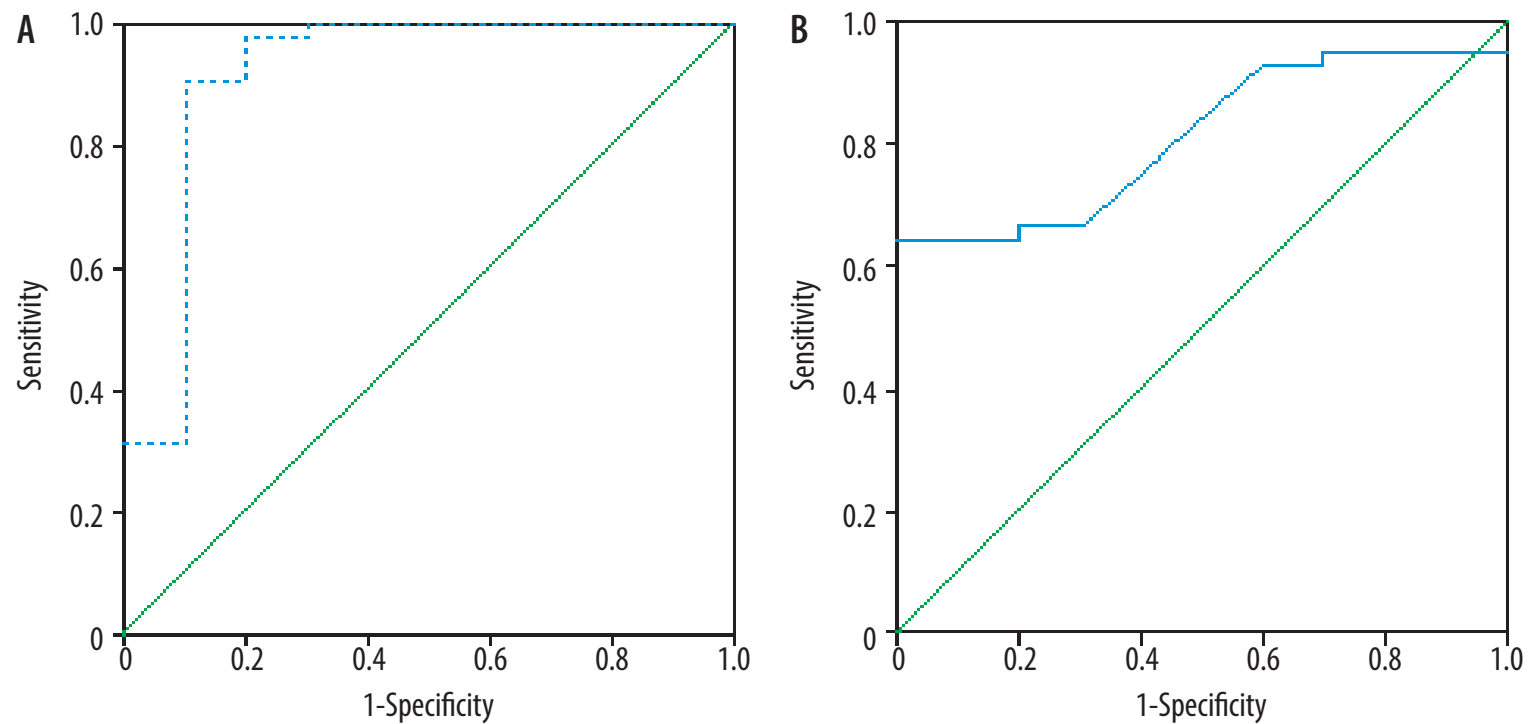

Figure 2. ROC curve of patients versus controls. A) The cutoff MD used to differentiate patients from controls was $1.50 \times 10^{-3} \mathrm{~mm}^{2} / \mathrm{s}$ with AUC of 0.919 , and accuracy of $94.2 \%$. B) The cutoff FA used to differentiate patients from controls was 0.31 with AUC of 0.813 , and accuracy of $67.3 \%$
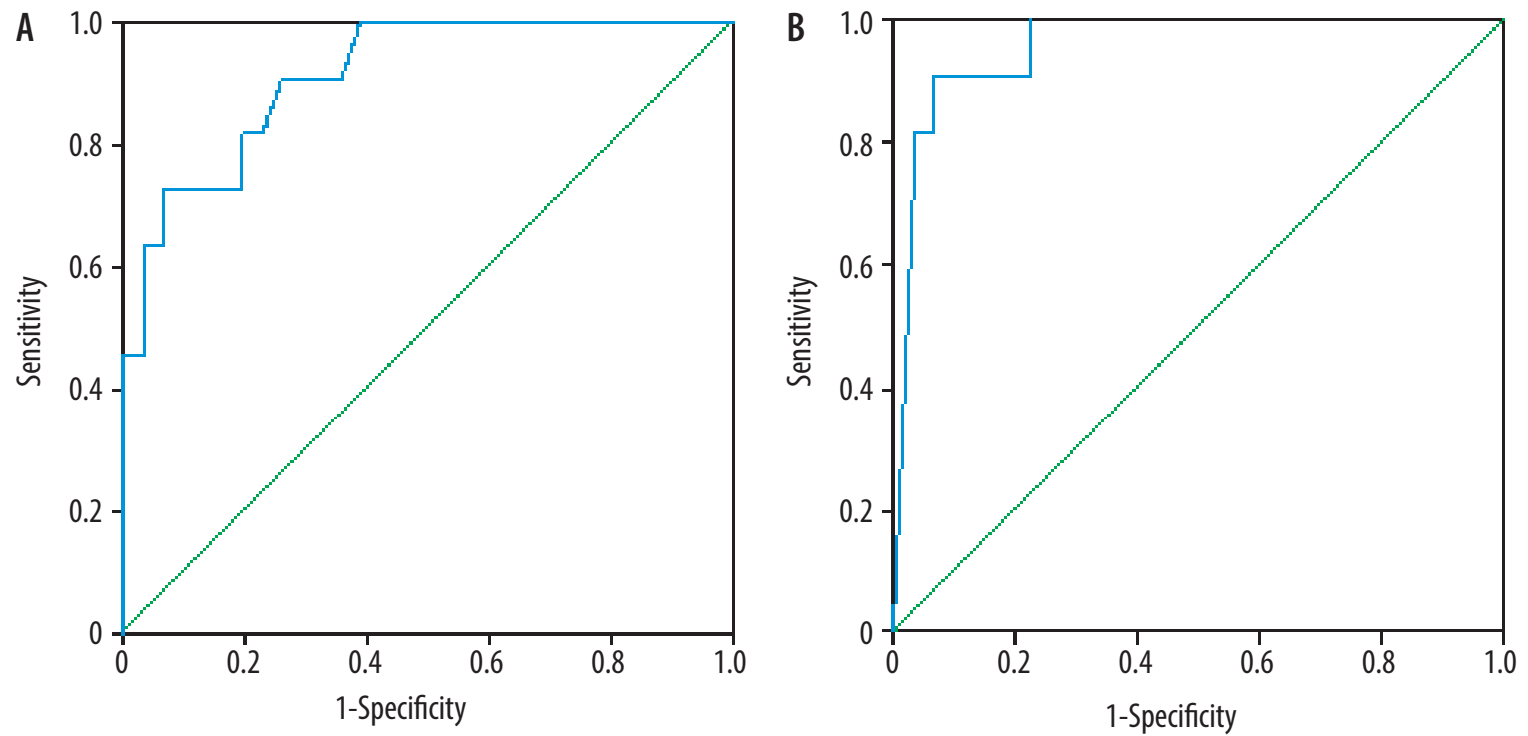

Figure 3. ROC curves of patients with AlH type I versus type II. A) The cutoff MD used to differentiate AlH type I from type II was $1.42 \times 10^{-3} \mathrm{~mm}^{2} / \mathrm{s}$ with AUC of 0.915 , and accuracy of $78.6 \%$. B) The cutoff FA used to differentiate AlH type I from type II was 0.42 with AUC of 0.959 , and accuracy of $80.9 \%$
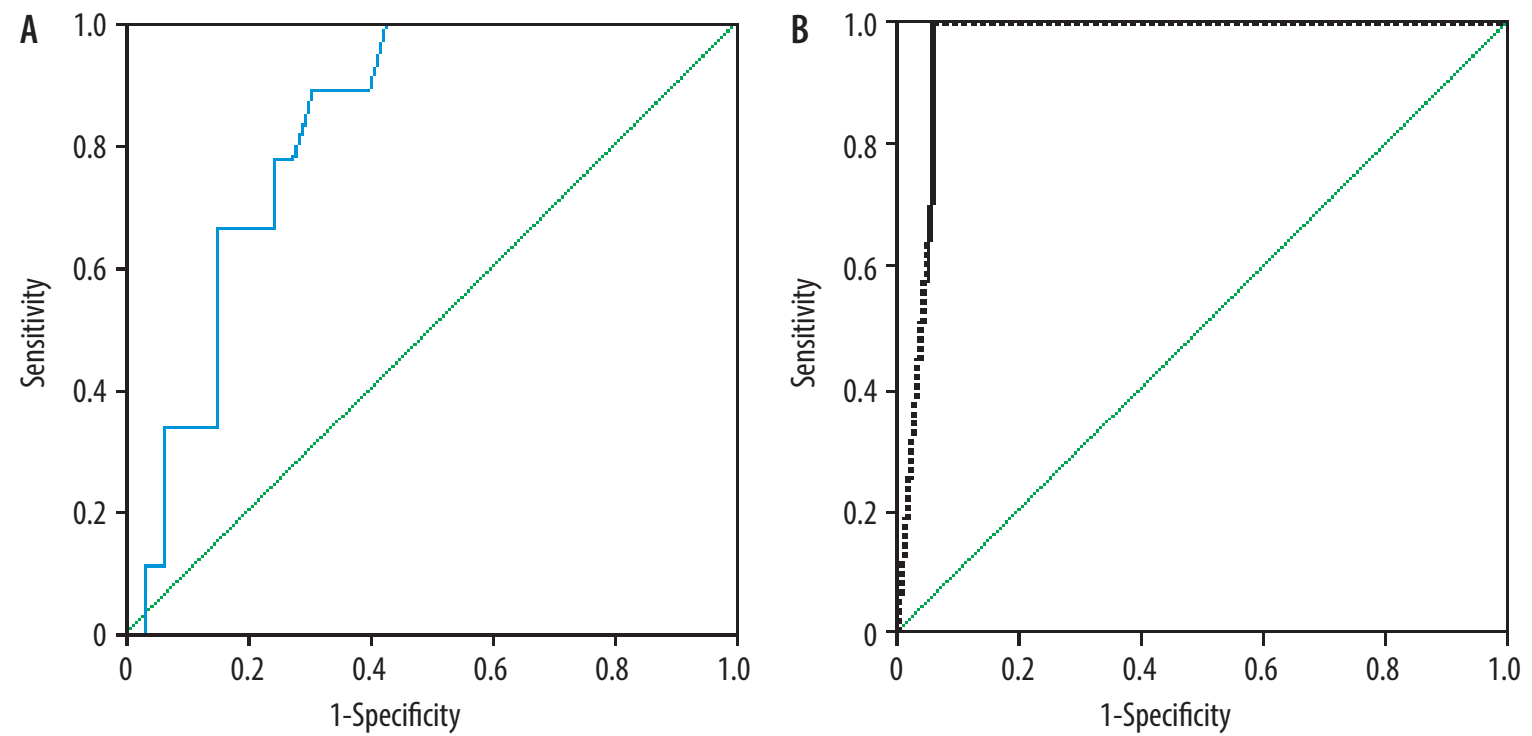

Figure 4. ROC curve results of patients with overlap syndrome versus without overlap syndrome. A) The cutoff MD and FA used to differentiate AlH with overlap syndrome from AlH without overlap syndrome was $1.42 \times 10^{-3} \mathrm{~mm}^{2} / \mathrm{s}$ with AUC of 0.828 , and accuracy of $73.8 \%$. B) The cutoff FA used to differentiate AlH with overlap syndrome from AlH without overlap syndrome was 0.50 with AUC of 0.963 , and accuracy of $92.9 \%$ 
interface hepatitis and rosette formation, and clinically ranging from asymptomatic to having liver failure. AIH is characterized by positive autoantibodies including ANA, ASMA, and anti-LKM1 and increased immunoglobulin G (IgG) [2-7].

In this study, the MD of the liver in children with AIH was significantly lower compared to the controls. This may be due to the presence of interface hepatitis at the portal-parenchymal interface, with dense plasma cell-rich lymphoplasmacytic, infiltrates, hepatocellular regeneration with rosette formation, emperipolesis (active penetration by one cell into and through a larger cell), and hepatocyte swelling and/or pycnotic necrosis piecemeal necrosis with periportal/periseptal lymphocyte infiltrating the portal plasma cells. Fibrosis is present in all children with AIH apart from those with the mildest forms of the disease [2-7].

One study reported that there is a statistical difference in the MD of children with hepatic fibrosis $(p=0.001)$ and controls, and within the different grades of hepatic fibrosis $(p=0.002)$, with correlation between the MD and fibrosis score $(r=0.807, p=0.001)$ [30]. Another study reported that the MD in children with fibrosis $\left(1.53 \pm 0.1710^{-3}\right.$ $\mathrm{mm}^{2} / \mathrm{s}$ ) is significantly lower than the MD of controls (1.74 $\pm 0.16^{\prime} 10^{-3} \mathrm{~mm}^{2} / \mathrm{s}$ ), and MD is negatively correlated with the different stages of fibrosis $(r=-0.799, p=0.001)$ and necro-inflammatory activity grade $(r=-0.468, p=0.001)$ [31]. Another study reported that hepatic MD can be used for differentiation of biliary atresia from non-biliary atresia in neonatal cholestasis, and a decreasing tends of MD with increasing degree of fibrosis [26]. The stretched exponential model shows a higher diagnostic performance for determining significant hepatic fibrosis than the monoexponential model of diffusion modules [28].

In this study, the FA is significantly higher in children with AIH than controls. This may be due to the increased connective tissue in the liver together with collagen fibre deposition, fatty infiltration, cell necrosis/apoptosis, and the presence of inflammatory cell infiltration. A previous study reported that the FA shows an increasing trend with increasing fibrotic stage, but there is an insignificant difference in the FA values $(p=0.183)$ at different fibrotic stages [26]. Another study added that the mean hepatic FA of hepatic parenchyma in neonates with biliary atresia $(0.34 \pm 0.04$ and $0.36 \pm 0.04)$ is significantly higher $(p=0.01,0.02)$ than that of Alagille syndrome $(0.30 \pm 0.06$ and $0.31 \pm 0.05)$ for both readers $(r=0.80, p=0.001)$ [29]
Based on the serum tests, there are 2 types of AIH; where AIH Type I is more common (two-thirds), tends to affect younger girls, and is usually associated with other autoimmune diseases and AIH type II (one-third), which primarily affects children between the ages of 2 and 14 years. While AIH generally occurs in early adulthood or adolescence, it can develop at any age. AIH represents approximately $10 \%$ of the 400 new paediatric referrals per year, two-thirds of the cases being AIH type I and onethird AIH type II [32]. In this study, AIH type II showed a lower MD and higher FA than AIH type I denoting that patients with AIH type II have more severe activity and fibrosis than type I.

Overlap syndrome is characterized by AIH with features of primary sclerosing cholangitis that are associated pathologically with a higher frequency of interface hepatitis $(p=0.008)$ and the presence of rosettes $(p=0.05)$ [30]. In this study, the MD was significantly lower in children with overlap syndrome. This may be attributed to the presence of associated pathological changes of the bile duct and the presence of cirrhotic changes [4-7]. This finding indicates that patients associated with the overlap syndrome have a more aggressive form of the disease.

Few studies have discussed the differences in DTI between 1.5 and 3.0 Tesla of the abdomen and spine. One study reported that the higher magnetic field strength provides a higher image quality in DTI of the spinal cord, better differentiation between the the renal cortex and medulla, and early detection of hepatic ischaemia-reperfusion injury [33-35].

There are some limitations to our study. First, there was a small number of patients, which limits the statistical power. Further studies are recommended upon a larger number of patients. Second, this study used DTI of the liver. Further studies using multiparametric MR imaging [36-43] combined with laboratory tests are recommended.

\section{Conclusions}

We conclude that DTI parameters can help in the diagnosis of AIH, detection of its phenotyping, and detection of the presence of associated overlap syndrome.

\section{Conflict of interest}

The authors report no conflict of interest.

\section{References}

1. Smolka V, Tkachyk O, Ehrmann J, et al. Acute onset of autoimmune hepatitis in children and adolescents. Hepatobiliary Pancreat Dis Int 2020; 19: 17-21.

2. Doycheva I, Watt KD, Gulamhusein AF. Autoimmune hepatitis: current and future therapeutic options. Liver Int 2019; 39: 1002-1013.
3. Tanaka A. Emerging novel treatments for autoimmune liver diseases. Hepatol Res 2019; 49: 489-499.

4. Porta G, Carvalho E, Santos JL, et al. Autoimmune hepatitis in 828 Brazilian children and adolescents: clinical and laboratory findings, histological profile, treatments, and outcomes. J Pediatr 2019; 95: 419-427. 
5. Mataya L, Patel N, Azzam RK. Autoimmune liver diseases in children. Pediatr Ann 2018; 47: e452-457.

6. Sokollik C, McLin VA, Vergani D, et al. Juvenile autoimmune hepatitis: a comprehensive review. J Autoimmun 2018; 95: 69-76.

7. Mieli-Vergani G, Vergani D, Baumann U, et al. Diagnosis and management of paediatric autoimmune liver disease: ESPGHAN hepatology committee position statement. J Pediatr Gastroenterol Nutr 2018; 66: 345-360.

8. Nastasio S, Sciveres M, Matarazzo L, et al. Old and new treatments for pediatric autoimmune hepatitis. Curr Pediatr Rev 2018; 14: 187-195.

9. Arcos-Machancoses JV, Molera Busoms C, Julio Tatis E, et al. Accuracy of the 2008 simplified criteria for the diagnosis of autoimmune hepatitis in children. Pediatr Gastroenterol Hepatol Nutr 2018; 21 : 118-126.

10. Malik N, Venkatesh SK. Imaging of autoimmune hepatitis and overlap syndromes. Abdom Radiol 2017; 42: 19-27.

11. Goertz RS, Gaßmann L, Strobel D, et al. Acoustic radiation force impulse (ARFI) elastography in autoimmune and cholestatic liver diseases. Ann Hepatol 2018; 18: 23-29.

12. Puustinen L, Hakkarainen A, Kivisaari R, et al. ${ }^{31}$ Phosphorus magnetic resonance spectroscopy of the liver for evaluating inflammation and fibrosis in autoimmune hepatitis. Scand J Gastroenterol 2017; 52: 886-892.

13. Wang J, Malik N, Yin M, et al. Magnetic resonance elastography is accurate in detecting advanced fibrosis in autoimmune hepatitis. World J Gastroenterol 2017; 23: 859-868.

14. Notohamiprodjo M, Dietrich O, Horger W, et al. Diffusion tensor imaging (DTI) of the kidney at 3 tesla-feasibility, protocol evaluation and comparison to 1.5 Tesla. Invest Radiol 2010; 45: 245-254.

15. Cheung JS, Fan SJ, Gao DS, et al. Diffusion tensor imaging of liver fibrosis in an experimental model. J Magn Reson Imaging 2010; 32: 1141-1148.

16. Huang M, Lu X, Wang X, et al. Diffusion tensor imaging quantifying the severity of chronic hepatitis in rats. BMC Med Imaging 2020; $20: 74$

17. Razek AAKA, Batouty N, Fathy W, et al. Diffusion tensor imaging of the optic disc in idiopathic intracranial hypertension. Neuroradiology 2018; 60: 1159-1166.

18. Razek AAKA, Al-Adlany MAAA, Alhadidy AM, et al. Diffusion tensor imaging of the renal cortex in diabetic patients: correlation with urinary and serum biomarkers. Abdom Radiol 2017; 42: 1493 1500 .

19. El-Serougy L, Abdel Razek AA, Ezzat A, et al. Assessment of diffusion tensor imaging metrics in differentiating low-grade from highgrade gliomas. Neuroradiol J 2016; 29: 400-407.

20. Khalek Abdel Razek AA. Characterization of salivary gland tumours with diffusion tensor imaging. Dentomaxillofac Radiol 2018;47: 20170343.

21. Razek AAKA. Diffusion tensor imaging in differentiation of residual head and neck squamous cell carcinoma from post-radiation changes. Magn Reson Imaging 2018; 54: 84-89.

22. Shenoy-Bhangle A, Baliyan V, Kordbacheh H, et al. Diffusion weighted magnetic resonance imaging of liver: Principles, clinical applications and recent updates. World J Hepatol 2017; 9: 1081-1091.
23. Razek AAKA, Abdalla A, Barakat T, et al. Assessment of the liver and spleen in children with Gaucher disease type I with diffusionweighted MR imaging. Blood Cells Mol Dis 2018; 68: 139-142.

24. Razek AA, Massoud SM, Azziz MR, et al. Prediction of esophageal varices in cirrhotic patients with apparent diffusion coefficient of the spleen. Abdom Imaging 2015; 40: 1465-1469.

25. Lee Y, Kim H. Assessment of diffusion tensor MR imaging [DTI] in liver fibrosis with minimal confounding effect of hepatic steatosis. Magn Reson Med 2015; 73: 1602-1608.

26. Liu B, Cai J, Zhu J, et al. Diffusion tensor imaging for evaluating biliary atresia in infants and neonates. PLoS One 2016; 11: e0168477.

27. Kim J, Yoon H, Lee MJ, et al. Clinical utility of mono-exponential model diffusion weighted imaging using two b-values compared to the bi- or stretched exponential model for the diagnosis of biliary atresia in infant liver MRI. PLoS One 2019; 14: e0226627.

28. Yoon H, Shin HJ, Kim MJ, et al. Quantitative imaging in pediatric hepatobiliary disease. Korean J Radiol 2019; 20: 1342-1357.

29. Abdel Razek AAK, Abdalla A, Elfar R, et al. Assessment of diffusion tensor imaging parameters of hepatic parenchyma for differentiation of biliary atresia from alagille syndrome. Korean J Radiol 2020; 21: $1367-1373$.

30. Razek AA, Abdalla A, Omran E, et al. Diagnosis and quantification of hepatic fibrosis in children with diffusion weighted MR imaging. Eur J Radiol 2011; 78: 129-134.

31. Razek AA, Khashaba M, Abdalla A, et al. Apparent diffusion coefficient value of hepatic fibrosis and inflammation in children with chronic hepatitis. Radiol Med 2014; 119: 903-909.

32. Singh H, Balouch F, Noble C, et al. Evolving practice and changing phenotype in pediatric autoimmune liver disease: outcomes from an Australian center. J Pediatr Gastroenterol Nutr 2018; 67: 80-85.

33. Rossi C, Boss A, Lindig TM, et al. Diffusion tensor imaging of the spinal cord at 1.5 and 3.0 Tesla. Rofo 2007; 179: 219-224.

34. Gürses B, Kiliçkesmez O, Taşdelen N, et al. Diffusion tensor imaging of the kidney at 3 Tesla MRI: normative values and repeatability of measurements in healthy volunteers. Diagn Interv Radiol 2011; 17: 317-322.

35. Cheung JS, Fan SJ, Chow AM, et al. In vivo DTI assessment of hepatic ischemia reperfusion injury in an experimental rat model. J Magn Reson Imaging 2009; 30: 890-895.

36. Abdel Razek AAK, Talaat M, El-Serougy L, et al. Clinical applications of arterial spin labeling in brain tumors. J Comput Assist Tomogr 2019; 43: 525-532.

37. Abdel Razek AAK, Talaat M, El-Serougy L, et al. Differentiating glioblastomas from solitary brain metastases using arterial spin labeling perfusion- and diffusion tensor imaging-derived metrics. World Neurosurg 2019; 127: e593-e598.

38. Abdel Razek A, Zaki M, Bayoumi D, et al. Diffusion tensor imaging parameters in differentiation recurrent breast cancer from postoperative changes in patients with breast-conserving surgery. Eur J Radiol 2019; 111: 76-80.

39. Razek AAA. Ashmalla G. Assessment of paraspinal neurogenic tumors with diffusion-weighted MR imaging. Eur Spine J 2018; 27 : 841-886.

40. Abdel Razek AA, Kamal E. Nasopharyngeal carcinoma: correlation of apparent diffusion coefficient value with prognostic parameters. Radiol Med 2013; 118: 534-539. 
41. Abdel Razek AA, Elkhamary S, Al-Mesfer S, et al. Correlation of apparent diffusion coefficient at $3 \mathrm{~T}$ with prognostic parameters of retinoblastoma. AJNR Am J Neuroradiol 2012; 33: 944-948.

42. Razek AA, Shabana AA, El Saied TO, et al. Diffusion tensor imaging of mild-moderate carpal tunnel syndrome: correlation with nerve conduction study and clinical tests. Clin Rheumatol 2017; 36: 2319-2324.
43. Abdel Razek AAK, El-Serougy L, Abdelsalam M, et al. Differentiation of primary central nervous system lymphoma from glioblastoma: quantitative analysis using arterial spin labeling and diffusion tensor imaging. World Neurosurg 2019; 123: e303-e309. 